\title{
Quantification of low-Z elements by energy-filtered scanning transmission electron microscopy
}

Saleh Firoozabadi ${ }^{1}$, Andreas Beyer ${ }^{2}$, Pirmin Kükelhan ${ }^{2}$, Damien Heimes ${ }^{2}$, Jannik Lehr ${ }^{3}$ and Kerstin Volz ${ }^{2}$

${ }^{1}$ Materials Science Centre and Department of Physics, Philipps University Marburg, Hans-Meerwein-Straße 6, Marburg, 35032, Germany, Marburg, Hessen, Germany, ${ }^{2}$ Materials Science Centre and Department of Physics, Philipps University Marburg, Hans-Meerwein-Straße 6, Marburg, 35032, Germany, Germany, ${ }^{3}$ Materials Science Centre and Department of Physics, Philipps University Marburg, Hans-Meerwein-Straße 6, Marburg, 35032, Germany, United States

One of the valuable tools established for the structural characterization of semiconductors is quantitative highangle annular dark-field (HAADF) scanning transmission electron microscopy (STEM). The combination of the so-called Z-contrast images and complimentary image simulations, considering only elastic scattering, allows composition determination [1]. Nevertheless, in the case of light elements, e.g., nitrogen-containing III/V semiconductors, high static atomic displacement (SAD) has a strong effect on the electron scattering angular dependence. It causes higher intensity at relatively low angles but hardly changes the intensity at high angular regimes [2]. There are sources of discrepancy between simulations and experiments especially at low angular regimes such as the influences of amorphous surface layers, inelastic scattering (plasmon excitation), phonon-correlation within the frozen-lattice approach, and distortions in the diffraction plane of the microscope $[3,4]$ which makes the composition determination of light elements disputable.

In this study, a method is established to determine the composition of low-Z elements on an atomic scale. As a model system, we choose GaNxAs1-x which is an interesting candidate in the field of optoelectronics due to its long wavelength. It is performed by comparison of annular dark-field STEM images and multislice image simulations at low angle regimes which are sensitive to nitrogen content. The sources of errors mentioned above are addressed as follows. We considered SAD by relaxing the supercells prior to multislice simulations. Energy filtered STEM is applied to reduce the effect of plasmon excitations. In the end, a fast pixelated detector is applied which leads to record the convergent beam electron diffraction pattern (CBED) for every scanning position. Here a flexible choice of angular range is accessible to minimize other sources of error.

Three GaNxAs1-x quantum well (QW) structures between GaAs barriers were grown via metalorganic vapor phase epitaxy (MOVPE) (Aixtron AIX 200 GFR, AIXTRON SE). Each QW layer has a width of $4.9 \mathrm{~nm}$ and the nitrogen content is about $2.7 \%$ determined by HRXRD. An electron transparent sample in [010] direction with defined thickness steps is prepared by focused ion beam (FIB) (JEOL JIB-4601F, JEOL Ltd.). Subsequently, the specimen is thinned with low voltage Ar ions using a NanoMill® (Model 1040, E. A. Fischione Instruments). For STEM investigations, a double aberration-corrected STEM JEOL JEM2200FS 
(JEOL Ltd.) operated at $200 \mathrm{kV}$ and equipped with an in-column Omega energy filter above a pixelated detector (pnCCD (S)TEM Camera, PNDetector) is employed. It allows obtaining energy-filtered (-6 to $6 \mathrm{eV}$ ) CBEDs for every probe position. To determine the QWs' composition, complementary image simulations were carried out by STEMsalabim software package [5] based on multislice image simulations [6]. GaNxAs1$\mathrm{x}$ supercells are created for the expected nitrogen compositions of $\mathrm{x}=0-0.1$ and relaxed by the valance force field model. The thermal diffuse scattering and chromatic aberration are also taken into account.

Facilitated by doing energy-filtered STEM in the optimum angular range, quantitative STEM at low angular regimes is possible. The determined thickness in the barrier area (GaAs) is in good agreement with the thickness measured by the well-established HAADF STEM method. Local intensity drops close to the interface of QWs can be seen which are caused by surface strain relaxation [7]. Although chains of nitrogen are visible in the nitrogen composition map, the composition profile and the width of QW show a good agreement with HRXRD results.

Financial support in the framework of SFB 1083 and GRK 1782 by the German Research Foundation (DFG) is greatly appreciated.

\section{References}

[1] LeBeau, J. M., Findlay, S. D., Allen, L. J., \& Stemmer, S. (2008). Quantitative atomic resolution scanning transmission electron microscopy. Physical Review Letters, 100(20), 206101.

[2] Müller-Caspary, K., Oppermann, O., Grieb, T., Krause, F. F., Rosenauer, A., Schowalter, M., ... \& Potapov, P. (2016). Materials characterisation by angle-resolved scanning transmission electron microscopy. Scientific reports, 6(1), 1-9.

[3] Beyer, A., Krause, F. F., Robert, H. L., Firoozabadi, S., Grieb, T., Kükelhan, P., ... \& Volz, K. (2020). Influence of plasmon excitations on atomic-resolution quantitative 4D scanning transmission electron microscopy. Scientific Reports, 10(1), 1-15.

[4] Grieb, T., Krause, F. F., Müller-Caspary, K., Firoozabadi, S., Mahr, C., Schowalter, M., ... \& Rosenauer, A. (2021). Angle-resolved STEM using an iris aperture: Scattering contributions and sources of error for the quantitative analysis in Si. Ultramicroscopy, 221, 113175.

[5] Oelerich, J. O., Duschek, L., Belz, J., Beyer, A., Baranovskii, S. D., \& Volz, K. (2017). STEMsalabim: a high-performance computing cluster friendly code for scanning transmission electron microscopy image simulations of thin specimens. Ultramicroscopy, 177, 91-96.

[6] Kirkland, E. J. (1998). Advanced Computing in Electron Microscopy Plenum Press. New York.

[7] Beyer, A., Duschek, L., Belz, J., Oelerich, J. O., Jandieri, K., \& Volz, K. (2017). Influence of surface relaxation of strained layers on atomic resolution ADF imaging. Ultramicroscopy, 181, 8-16. 